\title{
Special Issue: Localization in Wireless Sensor Networks
}

\author{
Slavisa Tomic ${ }^{1, *,+(\mathbb{D}) \text { and Marko Beko }}{ }^{1,2, *,+}$ \\ COPELABS, Universidade Lusófona de Humanidades e Tecnologias, 1749-024 Lisboa, Portugal \\ UNINOVA, Monte de Caparica, 2829-516 Caparica, Portugal \\ * Correspondence: slavisa.tomic@ulusofona.pt (S.T.); beko.marko@ulusofona.pt (M.B.); \\ Tel.: +351-217-515-500 (S.T.) \\ + All authors contributed equally to this work.
}

Received: 20 February 2020; Accepted: 25 February 2020; Published: 27 February 2020

\section{Introduction}

Recent and continuous development of mobile computing and user-centric applications has led to a requirement for accurate and low-cost localization and tracking systems. Global navigation satellite systems (GNSS), and in particular, global positioning system (GPS), have become part of our everyday lives and are commonly used for outdoor navigation and localization. However, most people spend a large amount of their time in indoor environments (home, office, school, etc.), where GPS has very limited or no functionality. Therefore, researchers have been exploring use of alternative sources of information for indoor localization.

With an objective of maintaining low implementation costs, making use of existing technologies, such as terrestrial radio frequency (RF) sources, when providing a solution to the localization problem is strongly encouraged. Amongst others, these include Wi-Fi, Bluetooth, and ultra-wide band (UWB) signals, but researchers also use infrared signals, images, acoustic, light, and magnetic fields to name a few. Nevertheless, the requirement for wide deployment of infrastructure has made these systems cost-ineffective for some time. Only recently, advances in RF and micro-electromechanical system integrated circuit design enabled the wide availability of low-cost sensors and permitted the use of large-scale networks with hundreds or even thousands of sensors, sensing at high spatial and temporal densities. Because of the immense network proportions, human moderation is almost impossible. Hence, in order to achieve autonomous network configuration and establish the quality of the network coverage and/or spatial/geographical relationship for signal analysis and data mining, automatic estimation of sensors' physical locations is of paramount importance. Moreover, localization using existing technologies is a key enabling technology for many applications that can improve safety and efficiency in everyday life, such as ambient assisted living (assistance for elderly or people with disabilities, smart parking, monitoring of storage conditions and goods, etc.), navigation (aid for visually impaired, guiding costumers through shopping malls, airports, etc.), and workforce management (finding nearby doctors or police officers), context-dependent information sharing, etc.), and is being actively pursued by technological giants, such as Google, Apple, Microsoft, and Huawei, as well as research groups in some of the leading universities worldwide. Nevertheless, current systems found in the literature provide unsatisfactory accuracies or require specific and expensive equipment that needs to be installed and maintained. Thus, the search for a robust and accurate localization system, applicable in real-time still remains open.

This Special Issue welcomed contributions that focus on novel solutions for target localization and tracking in wireless sensor networks (WSNs). In the Special Issue, we sought for adequately mature research works, assessed through analytical, simulation, or experimental models. Extensions of formerly published works were also allowed, as long as this fact was clearly stated within the submission and that the proposed extension was significantly novel. 


\section{Summary of Contributions}

This Special Issue represents a collection of unique contributions in the area of target localization in WSNs. Very important topics and issues were discussed and various directions for possible future research on the topic were recognized. In total, 13 authors have made valuable contribution to the Special Issue, coming from various regions around the world, such as Europe, Asia, North America, and Australia.

Published works in the Special Issue cover a wide range of topics, namely development of a prototype of a new cooperative unmanned aerial vehicles (UAV) swarm localization system using low-cost sensors and cameras [1], lightweight distributed localization schemes for energy-restricted WSNs [2], fabrication of additional (angle) information for aiding tracking performance [3], target localization using combined radio measurements in the presence of synchronization errors [4], performance comparison of existing algorithms for hyperbolic 3-dimensional localization [5], and characterization of the log-normal model for received signal strength (RSS) measurements [6]. A short overview of each of the above-mentioned works is provided in the following.

In [1], Goel et al. developed a novel cooperative localization prototype based on information sharing among UAVs and static anchor nodes for precise localization of the UAVs. The UAVs were retrofitted with low-cost sensors including a camera, GPS receiver, UWB radio, and low-cost inertial sensors. Performance of the proposed prototype was evaluated in real-world conditions in partially and obscured GNSS environments. The authors analyzed the performance in both centralized and distributed cooperative network designs. Their results showed that the developed system can achieve good accuracy (2-4 m) in partially GNSS denied environments, provided a consistent communication in the cooperative network is available. Moreover, Goel et al. provided an experimental validation to show that information sharing is beneficial to improve localization performance even in ideal GNSS environments. Their experiments indicated that the major challenges for low-cost cooperative networks are consistent connectivity among UAV platforms and sensor synchronization.

Xiong and Sichitiu proposed a new lightweight solution to bridge the gap between the high accuracy demand and low resources available for range-based localization in [2]. Their localization scheme is called KickLoc. It is a fully distributed scheme, which counts for the uncertainty of range measurements to minimize localization errors introduced from the distance measurement, and leverages information from all neighboring nodes for better location estimations. The authors performed extensive simulations in order to validate the feasibility, performance and robustness of the proposed system. They also implemented both the intuitive and the Kalman filter (KF) based algorithms on TI CC2530 ZigBee SoC and their customized acoustic platforms. The obtained results of such experiments indicate that KickLoc is able to provide consistent location estimation under different measurement models.

The work in [3] addressed the problem of tracking a moving target in non-line-of-sight (NLOS) environments based on range estimates extracted from RSS and time of arrival (TOA) measurements. The authors in [3] exploited the known architecture of reference points to act as an improper antenna array together with the range estimates in order to fabricate additional azimuth information of the signal emitted by the target to each reference point. Afterwards, they took advantage of azimuth estimates and converted the problem into a more convenient, polar space, where a linearization of the measurement models was achieved effortlessly. Finally, the derived linearized measurement model served as a building block on top of which prior knowledge acquired during the movement of the target is incorporated by adapting a (KF). The proposed approach was assessed via computer simulations in which a fairly complex target trajectory was studied. In all considered scenarios, the new approach exhibited good performance, corroborating the authors' intuition that there is some inherent information within the network topology that can be exploited in our advantage to enhance the performance of an algorithm.

Unlike most existing schemes where knowledge of the model parameters is postulated to reduce the high dimensionality of the cost functions involved in the location estimation process, Coluccia 
and Fascista took a different approach in [4]. Based on the fact that model parameters depend on the operational wireless context, and change over time due to presence of dynamic obstacles and other modification of the environment, they proposed a scheme that adaptively estimates them "on the field". One challenge that Coluccia and Fascista faced was the fact that joint maximum likelihood estimator (MLE) for the considered problem does not admit a closed-form solution, and that numerical optimization is virtually unfeasible due to the large number of nuisance parameters. To overcome these issues, they proposed a two-step algorithm that combines RSS and TOA measurements: (1) calibration phase exploits nodes at known locations to estimate the unknown RSS and TOA model parameters and (2) hybrid TOA/RSS range estimator is combined with an iterative least squares (LS) procedure to obtain the unknown target location. Numerical results in [4] indicate that the proposed algorithm works well, achieving almost the same localization accuracy of the joint MLE used as a theoretical benchmark.

In [5], Khalaf-Allah considered three closed-form LS algorithms, where two of them were adapted to exploit the knowledge about nuisance parameters for accurate three-dimensional localization based on time difference of arrival (TDOA) measurements. The algorithms utilized a single set an extended single set and a full set of the TDOA measurements. Their performance was validated through both simulations and real-world measurements, where the wireless system transmitters were placed in a quasi-coplanar arrangement. The performed analysis showed good corroboration between the results obtained via simulations and real-world measurements, exhibiting best performance for estimators using a single set of measurements in high signal-to-noise ratio (SNR) levels, and a full set of measurements at low SNR levels and total NLOS conditions. Therefore, Khalaf-Allah recommended using the full set of TDOA measurements for three-dimensional positioning in bad signal conditions, such as high noise levels and NLoS propagation.

Vallet García in [6] called attention to the fact that employing the classical RSS log-normal model in WSNs poses a series of challenges derived from (a) the model's structural limitations when it comes to explaining real observations, (b) the inherent hardware variability typically encountered in the low-cost nodes of WSNs, and (c) the inhomogeneity of the deployment environment. His main goal was to better characterize how these factors impact the model parameters, since this issue has received little attention in the literature. He qualitatively elaborated on their effects and interplay, and presented results of two quantitative empirical studies showing how much the parameters can vary depending on the nodes used in the model identification and their position in the environment, and the antenna directionality. He further showed that the path loss exponent and the reference power can be highly correlated and argued that real WSN deployments are better represented by random model parameters jointly accounting for hardware and local environmental characteristics, rather than by deterministic independent ones. Lastly, he concluded that taking this variability into account would result in more realistic models and plausible results derived from their usage.

\section{Conclusions}

This special issue features six exceptionally inspiring and top-quality works, addressing different aspects of the localization problem in WSNs. Some of these works addressed cooperative target localization problems, while others focused on non-cooperative localization. Practical settings, where model parameters were estimated, rather than assumed known, were also addressed, as well as real-time localization of a moving target. A set of real-world experimental measurements were performed in order to assess the performance of the proposed solutions. Even though different aspects of the problem of interest were addressed in individual works of this Special Issues, all of them are in agreement that target localization in WSNs is a very important practical problem which will continue to attract interest in both research and industrial societies in the following years.

Such reasoning is founded on the fact that we are living in a period of relentless and prompts development of new technologies (e.g., fifth generation and Internet of Things) and establishment of novel applications. We witnessed that employing localization technologies to complement and improve vehicular and pedestrian systems in the areas of intelligent transportation systems, robotics, 
location-based services, and automated vehicles have already begun. Additionally, we expect that the Internet of Things will not cease nurturing the growth of networks in their size and dynamics. Due to the augmented network size and node mobility, implementation of localization algorithms in a distributed manner is expected to become a requirement for many practical settings, such as smart buildings and smart cities.

Acknowledgments: The authors would like to express their sincere gratitude to Prof. Dharma P. Agrawal (Editor-in-Chief) for his kind invitation to guest-edit this Special Issue. Also, the authors are really grateful to the assistance of everyone in the editorial office, in particular the managing editor, Ms. Louise Liu. Moreover, without the cordial effort and professionalism of the anonymous reviewers, the Special Issue would not be able to achieve the quality that it did; thus, our special thanks to them. Lastly, we would like to thank all authors who have contributed to this Special Issue. Without their contributions, the Special Issue would not be possible.

Conflicts of Interest: The authors declare no conflict of interest.

\section{References}

1. Goel, S.; Kealy, A.; Lohani, B. Development and Experimental Evaluation of a Low-Cost Cooperative UAV Localization Network Prototype. J. Sens. Actuator Netw. 2018, 7, 42. [CrossRef]

2. Xiong, H.; Sichitiu, M.L. A Lightweight Localization Solution for Small, Low Resources WSNs. J. Sens. Actuator Netw. 2019, 8, 26. [CrossRef]

3. Tomic, S.; Beko, M.; Dinis, R.; Montezuma, P. Estimating Directional Data from Network Topology for Improving Tracking Performance. J. Sens. Actuator Netw. 2019, 8, 30. [CrossRef]

4. Coluccia, A.; Fascista, A. Hybrid TOA/RSS Range-Based Localization with Self-Calibration in Asynchronous Wireless Networks. J. Sens. Actuator Netw. 2019, 8, 31. [CrossRef]

5. Khalaf-Allah, M. Performance Comparison of Closed-Form Least Squares Algorithms for Hyperbolic 3-D Positioning. J. Sens. Actuator Netw. 2020, 9, 2. [CrossRef]

6. Vallet García, J.M. Characterization of the Log-Normal Model for Received Signal Strength Measurements in Real Wireless Sensor Networks. J. Sens. Actuator Netw. 2020, 9, 12. [CrossRef] 